\title{
Remodeling of hepatic vascular changes after specific chemotherapy of schistosomal periportal fibrosis
}

\author{
Zilton A Andrade, Ana Paula Baptista, Thaynã Souto Santana \\ Laboratório de Patologia Experimental, Centro de Pesquisas Gonçalo Moniz-Fiocruz, Rua Valdemar Falcão 121, \\ 40295-000 Salvador, BA, Brasil
}

\begin{abstract}
Hepatosplenic schistosomiasis was the first human disease in which the possibility of extensive long standing hepatic fibrosis being degraded and removed has been demonstrated. When such changes occurred, the main signs of portal hypertension (splenomegaly, esophageal varices) progressively disappeared, implying that a profound vascular remodeling was concomitantly occurring. Hepatic vascular alterations associated with advanced schistosomiasis have already been investigated. Obstruction of the intrahepatic portal vein branches, plus marked angiogenesis and compensatory hyperplasia and hypertrophy of the arterial tree are the main changes present. However, there are no data revealing how these vascular changes behave during the process of fibrosis regression. Here the mouse model of pipestem fibrosis was used in an investigation about these vascular alterations during the course of the infection, and also after treatment and cure of the disease. Animals representing the two polar hepatic forms of the infection were included: (1) "isolated granulomas" characterized by isolated periovular granulomas sparsely distributed throughout the hepatica parenchyma; and (2) 'pipestem fibrosis' with periovular granulomas and fibrosis being concentrated within portal spaces, before and after treatment, were studied by means of histological and vascular injection-corrosion techniques. Instances of widespread portal vein obstruction of several types were commonly found in the livers of the untreated animals. These obstructive lesions were soon repaired, and completely disappeared four months following specific treatment of schistosomiasis. Treatment was accomplished by the simultaneous administration of praziquantel and oxamniquine. The most impressive results were revealed by the technique of injection of colored masses into the portal system, followed by corrosion in strong acid. The vascular lesions of non-treated pipestem fibrosis were represented in the plastic casts by considerable diminution of the fine peripheral portal vein radicles, plus dilatation of periportal collaterals. Four months after treatment, this last picture appeared replaced by tufts of newly interwoven vessels formed along the main portal vein branches, disclosing a strong angiomatoid reparative change. Understanding about the cellular elements at play during fibrovascular repairing changes of hepatic schistosomiais represents a matter of considerable scientific and conceptual importance. At present time one may only speculate about the participation of some type of natural stem-cell capable of restoring the diseased liver back to normal once the cause of the disorder has been eliminated.
\end{abstract}

Key words: hepatic schistosomiasis - vascular changes - fibrosis reversion

Clinical (Bina \& Prata 1983, Dietze \& Prata 1986, Homeida et al. 1988, Mohamed-Ali et al. 1991) and pathological (Andrade et al. 1992) observations revealed that a proportion of patients with hepatosplenomegaly and portal hypertension due to Schistosoma mansoni infection, the so-called hepatosplenic schistosomiasis, could revert to normal, months or years after specific treatment. An instance of spontaneous regression of hepatosplenic schistosomiasis has even been observed in an isolated endemic area from the state of Minas Gerais, Brazil, probably due to the self-extinction of parasitism (Katz \& Brener 1966). Such observations were registered at a time when the concept about the irreversibility of hepatic fibrosis was taken for granted. Nowadays, such possibility is admitted while the cases of successful liver fibrosis rever-

Financial support: Fapesb, Pronex

${ }^{+}$Corresponding author: zilton@cpqgm.fiocruz.br

Received 25 May 2006

Accepted 26 June 2006 sion are being published (Geerts 2001, Desmet \& Roskams 2004), which included even cases of far advanced cirrhosis (Arthur 2002, Massarrat et al. 2004).

The crucial requirement is that the etiology of the fibrosis is identified and properly removed. On the other hand, studies regarding the cells and their soluble factors involved in fibrogenesis and fibrolysis, are becoming a matter of recent and intensive investigations (Bataller \& Brenner 2005).

Although the actual focus on fibrosis research is entirely justified, one must remember that fibrosis in the liver probably is not important by itself. The space usually occupied by fibrosis in most chronic liver diseases should not be sufficient enough to cause impact on the physiology of an organ with such considerable functional reserve. The significance of liver fibrosis is related to the fact that it usually serves as a supporting stroma for an abnormal vasculature. Vascular changes induced by severe previous insults are the source of physiologic disturbance, not only it is expressed as portal hypertension, but also as hepatic failure (Popper 1977). Thus, in schistosomiasis, portal vein obstructive lesions coupled to hepatic artery hypertrophy are the dominating vascular changes (Andrade \& Cheever 1977), while in cirrhosis a 
complex of porto-hepatic shunts, hepatic artery hypertrophy, and sinusoidal capillarization are responsible for the deranged physiology (Popper 1977). Usually these important vascular changes have not been particularly considered during the process of fibrosis regression.

The present investigation used the mouse model of schistosomal pipestem fibrosis (Warren 1966, Andrade \& Cheever 1993) in order to investigate on the behavior of the advanced vascular hepatic changes during the course of post-chemotherapy fibrosis regression.

\section{MATERIALS AND METHODS}

Animals and procedures - Fifty adult inbred Balb/c mice of both sexes were used. Their weighted around 18$20 \mathrm{~g}$ at the start of the experiment and were maintained in large metal boxes, with free access to a commercial balanced diet and water. Forty animals were submitted to infection with recently eliminated cercariae from the FS strain (Andrade \& Sadigursky 1985) of S. mansoni, by the transcutaneous route. Ten non-infected mice were kept in similar conditions. They were submitted to liver biopsy and histological examination, and to vascular injections. Two months after cercarial exposition all the animals were passing viable $S$. mansoni eggs in the stools. Two months later (16 weeks of infection) the cumulative mortality among the infected animals was found to be rather unexpected and high. The surviving animals were submitted to a liver biopsy and 12 of them were selected after microscopic examination to be treated some days later with both oxamniquine and praziquantel. After treatment no further mortality occurred. Also, no non-infected controls died. But the infected and non-treated mice all died during the following four months. The drugs were administered by gavage. Each animal received the equivalent of $100 \mathrm{mg}$ per kg of body weight of oxamniquine. The following day praziquantel was administered to the same animals, the dose of $400 \mathrm{mg} / \mathrm{kg} / \mathrm{bw}$ being divided into two halves, one given at morning and the other in the afternoon. Four months after treatment the animals were killed, and the data obtained by histopathology were compared with those observed in the biopsy material from the same animal. The vascular casts obtained after treatment where compared with those from normal mice. Due to the high mortality of the animals, the vascular casts of the portal system could not be obtained from untreated mice with "pipestem" fibrosis. However, whenever necessary comparisons were made with material obtained from previous experiments, with the casts kept in our files (Andrade 1987).

Liver biopsy - Under sterile conditions and sodium pentobarbital general anesthesia the skin of the abdomen was shaved and cleaned. A small incision was made at the abdominal middle line and through it part of a liver lobe was exposed, tied and removed. The wound was closed with separate sutures.

Histopathology - Fragments of the liver taken by biopsy (just before treatment) and by necropsy (four months after treatment) were fixed in $10 \%$ neutral formalin and routinely embedded in paraffin. Sections were stained with hematoxilyn and eosin, with Sirius-red for collagen and orcein for elastic tissue.
Vascular injections - Two livers taken from normal non-infected mice, and seven from the infected and treated mice were submitted to vascular injection-corrosion studies. The portal system was first flushed from the portal vein trunk with saline to clear it from blood. This procedure was immediately followed by injection of $14 \%$ acetone solution of vinyl chloride-acetate (Union Carbide Plastics Co. grade VYHH, US), colored with carmine. The ininjection was made manually with a $10 \mathrm{cc}$ glass syringe until an uniform filling of the portal system was achieved. After injection, the liver was removed and kept in water overnight at room temperature. Then, the liver tissue was digested in a 50\% hydrochloric acid solution. One week later the plastic cast was taken out of the acid, washed several times in water and alcohol, dried, and examined directly with the naked eye and under a dissecting microscope. The plastic casts were analyzed using a SZX-9 Olympus stereoscopic microscope coupled to a DP-12 Olympus digital camera.

\section{RESULTS}

The liver fragments obtained by surgical biopsy taken at 16 weeks of infection revealed numerous periovular granulomas concentrated within periportal spaces, which appeared amplified by fibrosis up to the point to make connections with neighboring spaces, sometimes fusing some of them into large and irregular fibrotic areas. In between the areas of fibrosis the liver parenchyma appeared with its normal architecture. Obstructive vascular changes were detected in the large and medium-sized branches of the intrahepatic portal veins, especially evident in orcein-elastic stained slides (Fig. 1). The vessel walls and their lumina were partially or totally replaced by inflammatory and reparative tissue, sometimes centered by viable and degraded schistosome eggs, some of them showing evidence of calcification. The limiting elastic membrane of the portal veins appeared frequently fragmented and duplicated, forming irregular concentrations of fragmented elastic fibers in several focal areas around the most damaged vessels. In all sections examined, partial and total vascular obstruction was disclosed to be present in every portal space, both small and large. The tissue filling the vascular lumen seemed a proliferating connective tissue, formed by fusiform cells mixed with macrophages, lymphocytes and a few polymorphonuclear eosinophils, the numerous vessels appearing as clefts. When such intra-vascular tissue seemed more mature, it simulated an organized thrombus. The small-sized thinwalled blood vessels were numerous, represented by slits or clefts in the middle of the fibrotic-inflammatory changes of the portal spaces.

Four months after specific chemotherapy the picture changed considerably. No evidence of vascular obstruction was found in the several sections examined. The vessels appeared numerous, dilated, congested and thinwalled. Portal fibrosis diminished in extent and density, the fibrous tissue appearing as a loose, edematous tissue, containing calcified egg remnants, focal pigmentation, and a few scattered inflammatory cells (Fig. 2A). Collagen was seen in Sirius staining slides as woven and fragmented fibers, with focal areas of dissolution. The distribution of elastic fibers in the vascular walls varied 

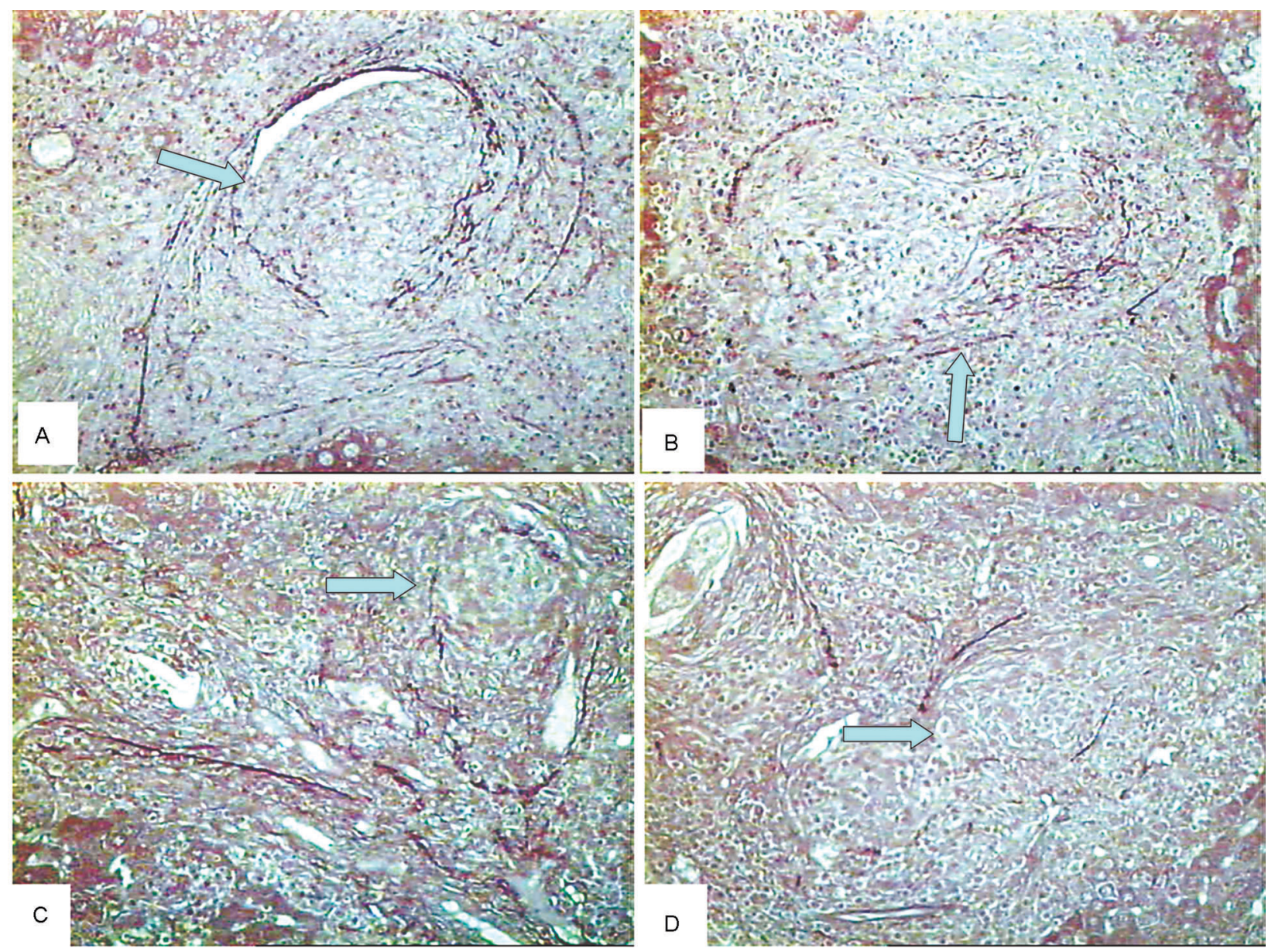

Fig. 1: periportal vascular obstructive lesions present in the liver of mice with non-treated 4-month old Schistosoma mansoni infection. Arrows point to the presence of fibrotic and inflammatory tissue filling the lumen of portal vessels. Orcein stain for elastic tissue, $\times 400$.

from one vassel to the next, even in a same vessel, revealing areas where the electic tissue was absent, side by side with other areas with elastic duplication or even evident hyperplasia. Proliferation of endothelial cells was evident, even in routinely stained slides, sometimes forming bridges inside the vascular lumen (Fig. 3D).

Vascular casts - Mice with periportal "pipestem" fibrosis due to schistosomiasis presented considerable decrease of the smaller ramifications of the vascular tree when compared with those obtained from the normal (Fig. 3 ). The smaller vessels are not observed in the infected animals, while the large ones (from the 1st, 2nd, and 3rd order dichotomization) exhibit numerous short spikes, which give a characteristic hairy appearance to the casts (Fig. 3B). Besides the amputation of the smaller vessels, the remaining portal vessels seen in the casts from infected animals also present frequent alterations, such as tortuosities, sudden caliber reduction, abnormal anastomosis with neighboring vessels or within the same vessels (short circuits).

The vascular casts obtained from infected mice four months after specific treatment showed and abundance of small ramifications sprouting from the larger branches of the portal system. The aspect was that of a growing tree in the spring (Fig 3C, D). The irregularities observed in the larger vessels of the infected mice were not observed any more when a careful examination of the casts was made under the lupa.

\section{DISCUSSION}

Present results reveal that the cure of advanced murine schistosomisis is not only followed by fibrosis regression, but also by a considerable vascular remodeling. These results indicate that, besides the interplay of factors already pointed out by connective tissue research, such as metalloproteinases, TIMPS, cells, and cytokines on extracellular matrix degradation, there are even more complex factors involved in hepatic fibrosis regression. That is indicated by the impressive vascular remodeling herewith observed, which involved the repair of the damaged vascular walls of the portal system that resulted in the opening of obstructed vessels, and the modifications detected on their walls, suggestive of a process of vascular cleaning and repairing. Furthermore, the sprouting of numerous new vessels suggested an important role being played by angiogenesis. The process of angiogenesis has more recently been linked with fibrosis formation 

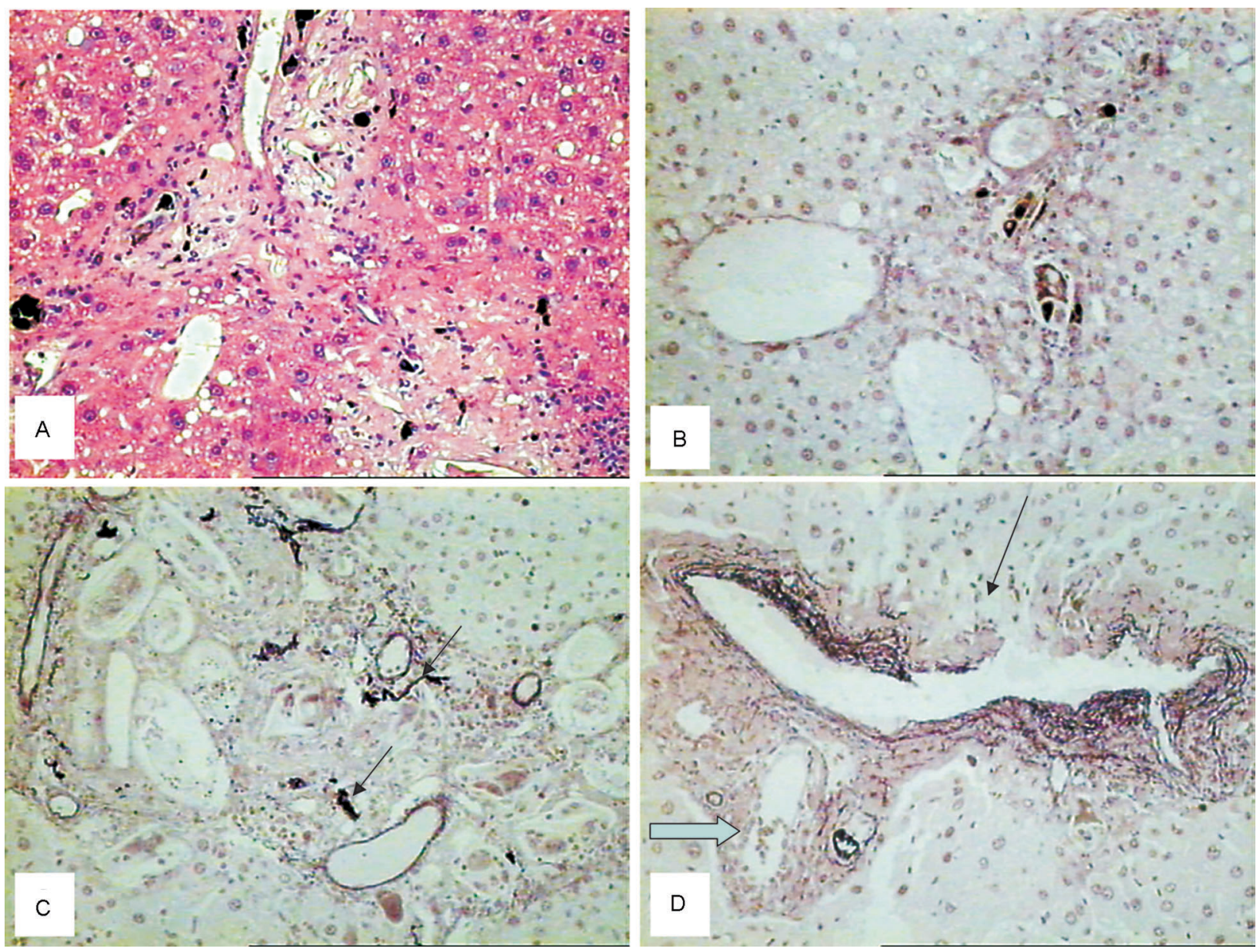

Fig. 2: aspects of the periportal tissue of mice that had previously been proved to present schistosomal periportal ("pipestem") fibrosis, now observed four months after specific treatment of schistosomiasis. A: periportal fibrosis is minimal and disclose a loose, edematous structure. The black dots represent calcified eggs and schistosomal pigment; B: portal vessels now appear with wide open lumina and thinwalled walls. C: remnants of broken elastic fibers are scattered amongst dilated thin-walled portal veins and a few arteries (arrows); D: a large portal vein showing an irregular distribution of elastic tissue in its wall, probably being remodeled, with one area where the fibers are absent (thin arrow). In the vessels seen below proliferating endothelial cells form a bridge within the lumen of a portal vein (arrow). Orcein elastic stain. All pictures with a $\times 200$ magnification.

(Rosmoduc et al. 1999, Bataller \& Brenner 2005), rather than with fibrosis removal. But present data are consistent with the possibility of angiogenesis being able to play a two-way role. Although the presence of a rich vasculature in schistosomiasis treated mice could be explained by re-canalization of large obstructed portal vessels and pressure dilatation of the numerous small branches and capillaries, the vascular neo-formation (angiogenesis) cannot be discarded. The mechanisms behind these changes seem to have a high degree of complexity. One can speculate about the presence of putative stem-cells, resident or migrated from elsewhere, with potential to induce vascular remodeling, removal of the excess extracellular matrix, and angiogenesis (Theise 2003). Recently, great emphasis has been given to angiogenesis in fibrosis formation (Rosmoduc et al. 1999, Souza 2003). The evidences are so strong on this regard that a recent editorial suggests that new approaches to therapy of liver fibrosis may be derived from progress on angiogenesis manage- ment. (Lai \& Adams 2005). Now, the evidences of endothelial proliferation and the appearance of numerous vessels in periportal tissue following curative chemotherapy of schistosomiais bring about the question whether angiogenesis may act in a two-way mechanism, being important to form as well as to remove fibrosis. Future research is expected to clarify this matter.

\section{REFERENCES}

Andrade ZA 1987. Pathogenesis of pipe-stem fibrosis of the liver (experimental observation on murine schistosomiasis). Mem Inst Oswaldo Cruz 82: 325-334.

Andrade ZA, Cheever AW 1993. Characterization of the murine model of schistosomal hepatic periportal fibrosis ("pipestem" fibrosis). Internat J Exper Pathol 74: 195-202.

Andrade ZA, Peixoto E, Guerret S, Grimaud JÁ 1992. Hepatic connective tissue changes in hepatosplenic schistosomiasis. Hum Pathol 23: 566-573. 


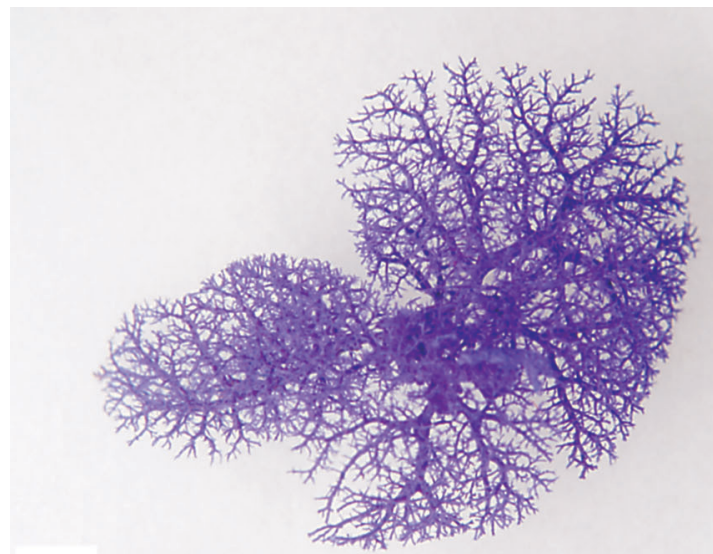

A
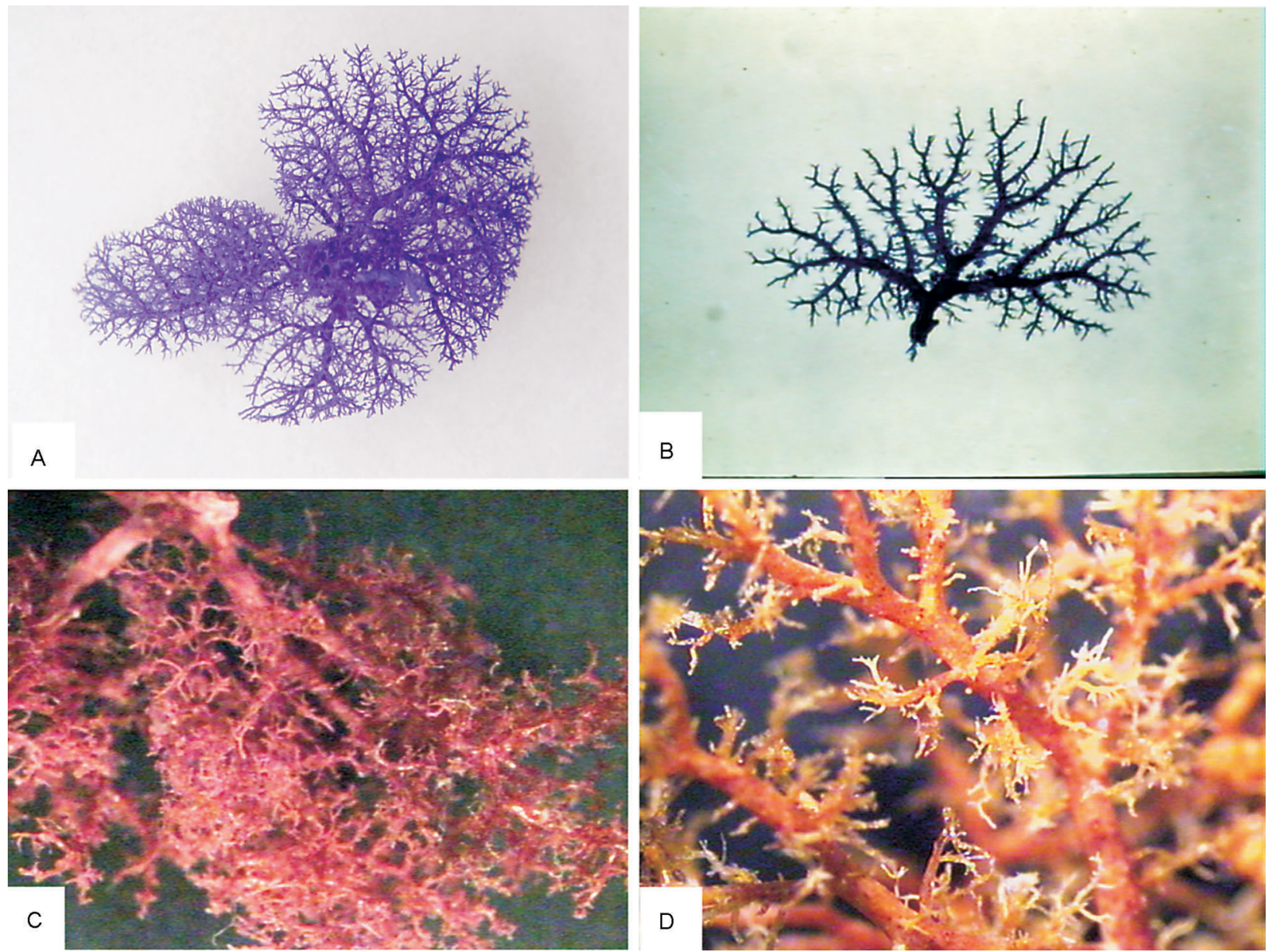

Fig. 3: casts of vinyl chloride-acetate obtained from the portal system of mice. A: normal mouse; B: mice with chronic schistosomiasis showing severe amputation of the smaller branches, and short spikes arising in right angles from the major portal branches (Andrade 1987); $\mathrm{C}$ and D: four months after treatment of chronic schistosomiasis. Sprouting of numerous small vessels imitating a picture of a growing tree in the spring..

Andrade ZA, Sadigursky M 1985. Um estudo comparativo das cepas Feira de Santana (Bahia) e Porto Rico do Schistosoma mansoni na infecção experimental do camundongo. Mem Inst Oswaldo Cruz 80: 37-40.

Arthur MJP 2002. Reversibility of liver fibrosis and cirrhosis following treatment for hepatitis C (Editorial). Gastroenterology 122: 1525-1528.

Bataller R, Brenner DA 2005. Liver fibrosis. J Clin Invest 115: 209-218.

Bina JC, Prata A 1983. Regressão da hepatoesplenomegalia pelo tratamento específico da esquistossomose. Rev Soc Bras Med Trop 16: 213-218.

Desmet VJ, Roskams T 2004. Cirrhosis reversal: a duel between dogma and myth. J Hepatol 40: 860-867.

Dietze RS, Prata A 1986. Rate of reversion of hepatosplenic schistosomiasis after specific chemotherapy. Rev Soc Bras Med Trop 19: 69-73.

Geerts A 2001. History, heterogeneity, developmental biology, and functions of quiescent hepatic stellate cells. Sem Liver Dis 21: 311-335.

Homeida MA, Ahmed S, Dafalla A, Sulliman S, Eltom I, Nash T, Bennett JL 1988. Morbidity associated with Schisto- soma mansoni infection as determined by ultrasound: a study in Gezira, Sudan. Am J Trop Med Hyg 39: 196-201.

Katz N, Brener Z 1966. Evolução clínica de 112 casos de esquistossomose mansoni observados após dez anos de permanência em focos endêmicos de Minas Gerais. Rev Inst Med Trop São Paulo 8: 139-142.

Lai WK, Adams DH 2005. Angiogenesis and chronic inflammation; the potencial for novel therapeutic approaches in chronic liver diseases (Editorial). J Hepatol 42: 7-11.

Massarrat S, Fallahazad V, Kamalian N 2004. Clinical, biochemical and imaging-verified regression of hepatitis B-induced cirrhosis. Liver Intern 24: 105-109.

Mohamed-Ali Q, Doehring-Schwerdtfeger E, Abdel-Rahim IM, Schlake J, Kardoff R, Franke D, Kaiser C, Elsheikh M, Abdalla S, Schafer P, Ehrich JHH 1991. Ultrasonographic investigation of periportal fibrosis in children with Schistosoma mansoni infection: reversibility of morbidity seven months after treatment with praziquantel. Am J Trop Med Hyg 44: 444-451.

Popper H 1977. Pathologic aspects of cirrhosis. A Review. Am J Pathol 87: 227-264.

Rosmorduc O, Wendum D, Corpechot C, Galy B, Sebbag N, 
Raleigh J, Housset C, Poupon R 1999. Hepatocellular hypoxia-induced vascular endothelial growth factor expression and angiogenesis in experimental biliary cirrhosis. Am J Pathol 155: 1065-1073.

Souza MM 2003. Estudos sobre a Fibrose Septal Hepática Induzida por Capillaria Hepatica (Patogênese e Evolução), $\mathrm{PhD}$ Thesis, Curso de Pós Graduação em Patologia Ex- perimental, UFBA, Salvador.

Theise ND 2003. Liver stem cells: the fall and rise of tissue biology (Editorial). Hepatology 38: 804-806.

Warren KS 1966. The pathogenesis of "clay-pipe stem cirrhosis" in mice with chronic schistosomiasis mansoni, with a note on the longevity of the schistosomes. Am J Pathol 49: 477-489. 